\title{
Major Abdominal Surgery, Neurosurgery, Orthopedic Surgery in Children aged between 10 and 18 years and Postoperative Outcome
}

Claudine Kumba ( $\sim$ claudine.kumba@gmail.com)

Hôpital Universitaire Necker Enfants Malades, Assistance Publique Hôpitaux de Paris, APHP, Université de Paris https://orcid.org/0000-0002-9748-5141

\section{Research Article}

Keywords: Children, abdominal surgery, neurosurgery, orthopedics, outcome

Posted Date: June 29th, 2021

DOI: https://doi.org/10.21203/rs.3.rs-666955/v1

License: @) (i) This work is licensed under a Creative Commons Attribution 4.0 International License. Read Full License

Version of Record: A version of this preprint was published at SOJ Pediatrics and Clinical Neonatology on September 1st, 2021. See the published version at https://doi.org/10.53902/SOJPCN.2021.01.000509. 


\section{Abstract}

Background

In a previously conducted monocentric retrospective study, predictors of postoperative outcome in children included American Society of Anesthesiologists score, transfusion, emergency interventions, surgery and age. A study has been undertaken to describe postoperative outcome in patients aged between 10 and 18 years included in the initial study in abdominal surgery, neurosurgery and orthopedics.

$\underline{\text { Aims }}$

To describe postoperative outcome in children aged between 10 and 18 years.

\section{Methods}

Secondary analysis of postoperative outcome in children aged between 10 and 18 years included retrospectively in the initial study of 594 patients. The study was approved by the Ethics Committee.

$\underline{\text { Results }}$

There were 221 children with a mean age of $165.5 \pm 24.3$ months in abdominal surgery, neurosurgery and orthopedics. $16.3 \%$ patients had intra-operative or postoperative complications. Intra-operatively, $3.2 \%$ had hemorrhagic shock, $0.45 \%$ anaphylaxis and $0.45 \%$ respiratory failure. Postoperatively, $3.2 \%$ had neurologic failure, $2.3 \%$ respiratory failure, $0.45 \%$ cardio-circulatory failure, $0.45 \%$ endocrinal failure, $0.45 \%$ had hepatic failure, $0.45 \%$ multiple organ failure, and $0.45 \%$ had renal failure. In-hospital mortality rate was $0.45 \%$.

\section{Conclusion}

These results emphasize the importance of optimizing intra-operative management in critically ill patients in major surgery with goal directed therapies in order to improve postoperative outcome.

\section{Keynotes}

Postoperative outcome is multifactorial in surgical patients. Intra-operative goal directed fluid and hemodynamic therapy to optimize patient management with validated tools in children is one of the main aims of goal directed therapies which have the objective to improve postoperative outcome in surgical patients. It is time to integrate goal directed therapies in our routine practice for intraoperative management to improve postoperative outcome in children.

\section{Introduction}

A monocentric retrospective study was conducted in 594 patients with a mean age of $90.86 \pm 71.80$ months with the objective to determine predictors of postoperative outcome in abdominal surgery, neurosurgery and orthopedics (1). Postoperative outcome predictors in this study were American Society of Anesthesiologists status (ASA), transfusion, emergency, surgery and age. A similar analysis in different surgical settings was undertaken and conducted to the same conclusions $(2,3,4)$.

Postoperative outcome was defined as organ failure, infections, re-operations, length of stay in the intensive care unit, length of hospital stay, total length of hospital stay and length of mechanical ventilation. Meta-analyses in patients under 18 years revealed that intra-operative goal directed fluid and hemodynamic therapy is not a routine generalized practice, that non optimal intraoperative hemodynamic parameters were predictors of adverse postoperative outcome in terms of morbidity and mortality, that transfusion goal directed therapy with point of care tests reduced fresh frozen plasma transfusion and length of hospital stay and that enhanced recovery after surgery applied in surgical settings reduced postoperative complications $(5,6,7)$.

To emphasize the importance of postoperative outcome in surgical children, a secondary analysis 
was conducted with the objective to describe in detail with regard to age, postoperative evolution in children aged between 10 and 18 years included in the initial retrospective study (1). Similar secondary analyses have been conducted in pre-terms, infants less than 1 year old, children aged between 1 and 3 years, children from 3 to 6 years old and children from 6 to 10 years with the objective to describe postoperative outcome with consideration to age since age has been revealed to be an independent predictor of postoperative outcome $(1,2,3,4)$.

\section{Methods And Materials}

Secondary analysis of children between 10 and 18 years old included in the initial study (1).

The study was declared to the CNIL, National Commission for Computer Science and Liberties on 21 February 2017 under the registration number 2028257 v0. The Ethics Committee of Necker approved the study on 21 March 2017 under the registration number 2017-CK-5-R1. Patients were included retrospectively from 1 January 2014 to 17 May 2017. Inclusion criteria were children aged between 10 and 18 years old.

Exclusion criteria were children aged less than 10 years old and older than 18 years. Statistics were analyzed with XLSTAT 2020.4.1 software.

Continuous variables were described in means \pm standard deviation or medians with interquartile ranges. Categorial variables were described in proportions.

\section{Results}

Table 1 illustrates general characteristics.

There were 221 children with a mean age of $165.5 \pm 24.3$ months.

There were 38 patients $(17.2 \%)$ in abdominal surgery, $65(29.4 \%)$ in neurosurgery and $118(53.4 \%)$ in

orthopedics. 25 patients (11.3\%) had an emergency surgery. 121 patients (54.8\%) were ASA 3 and 19

(8.6\%) were ASA 4.

Table 2 illustrates types of surgery.

The most common surgical interventions were scoliosis in 86 patients (39.9\%), intracerebral tumor

resection in 28 patients $(12.7 \%)$, limb tumor resection in 19 patients $(8.6 \%)$, pelvic tumor in 10

patients (4.5\%), renal transplantation in 8 patients (3.6\%), epileptogenic lesion resection in 7 
patients (3.2\%), craniosynostosis in 5 patients (2.3\%), decompressive craniectomy in 5 patients

(2.3\%), femoral osteotomy in 5 patients (2.3\%), Chiari's malformation in 4 patients $(1.8 \%)$, liver

transplantation in 4 patients (1.8\%), ventriculostomy in 4 patients (1.8\%) and limb amputation in 3

patients $(1.4 \%)$.

36 patients (16.3\%) had intra-operative and or postoperative complications (organ failure or sepsis).

7 patients (3.2\%) had intra-operative hemorrhagic shock, $1(0.45 \%)$ had intra-operative anaphylaxis

and $1(0.45 \%)$ had intra-operative respiratory failure. 7 patients $(3.2 \%)$ had postoperative neurologic

failure, $5(2.3 \%)$ had postoperative respiratory failure, $1(0.45 \%)$ had postoperative cardio-circulatory

failure, $1(0.45 \%)$ had postoperative endocrinal failure, $1(0.45 \%)$ had postoperative hepatic failure,

$1(0.45 \%)$ had postoperative multiple organ failure, and $1(0.45 \%)$ had postoperative renal failure. The

most common postoperative infections were surgical wound sepsis in 7 patients (3.2\%), abdominal

sepsis in $5(2.3 \%)$, septicemia in $3(1.4 \%)$, urinary sepsis in $3(1.4 \%)$, neuro-meningeal sepsis in 1

(0.45\%) and pulmonary sepsis in $1(0.45 \%)$.

15 patients $(6.8 \%)$ had re-operations.

83 patients (37.6\%) had intra-operative transfusion. Mean preoperative and postoperative

hemoglobin levels were $12.4 \pm 1.7 \mathrm{~g} / \mathrm{dL}$ and $11.4 \pm 1.7 \mathrm{~g} / \mathrm{dL}$ respectively.

In-hospital mortality rate was $0.45 \%$ in 1 trauma patient who underwent a decompressive

craniectomy and presented postoperative multiple organ failure and died on postoperative day 7 
(table 3).

Median total length of hospital stay was 9 days[5-18].

Table 4 illustrates outcomes per surgery.

Table 5 illustrates co-morbidities.

The most common co-morbidities were intracerebral tumor in 27 patients (12.2\%), cerebral anoxic

lesions 19 patients (8.6\%), epilepsy 14 patients (6.3\%), Ewing's sarcoma 12 patients (5.4\%), cancer 9

patients $(4.1 \%)$, neurofibromatosis 8 patients $(3.6 \%)$, chronic renal failure 8 patients $(3.6 \%)$, Chiari's

malformation 6 patients (2.7\%), hepatic failure 5 patients $(2.2 \%)$ and psychomotor deficiency 4

patients $(1.8 \%)$.

\section{Discussion And Conclusion}

In this cohort of 221 patients aged between 10 and 18 years in major abdominal surgery, neurosurgery and orthopedic surgery, $16.3 \%$ of the patients presented with intra-operative and or postoperative complications and most were ASA grade $\geq 3$. The most common intra-operative complication was hemorrhagic shock and $37.6 \%$ were transfused intra-operatively. Transfusion goal directed protocols with point of care tests should be integrated in intra-operative management in hemorrhagic surgery with the aim to optimize patient blood administration in children. A recent meta-analysis in children aged less than 18 years old in hemorrhagic settings revealed that transfusion guided with point care tests reduced fresh frozen plasma administration and length of hospital stay (5). Since transfusion is one of the predictors of postoperative outcome $(1,2,3,4)$ it is mandatory to have patient blood management protocols in hemorrhagic surgery since these protocols are not yet a routine generalized practice in our Hospital. Length of hospital stay as well as hospital costs were significantly higher in transfused patients than in non-transfused patients (8). The rate of patients with postoperative complications in this study emphasizes the importance of optimizing intra-operative patient management in critically ill patients in major surgery $(5,6,7,8,9,10,11,12,13,14)$. Optimizing intra-operative patient management with goal directed therapies is not a routine generalized practice in our Hospital. Intra-operative goal directed therapies include goal directed fluid and hemodynamic therapy with validated tools in children, transfusion goal directed protocols with point of care tests and enhanced recovery after surgery 
$(5,6,7,8,9,10,11,12,13,14)$. The aim of goal directed therapies is to optimize patient intra-operative

management with the objective to improve postoperative outcome which depends on multiple

factors. Non optimal intra-operative hemodynamic parameters have been correlated to adverse

postoperative outcome in surgical pediatric patients (6). Considering the results of previous studies

there is enough evidence to integrate goal directed therapies for intra-operative patient

management in pediatric surgical patients to optimize postoperative outcome $(1,2,3,4,5,6,7,8)$.

\section{Declarations}

Conflicts of Interest The author declared no conflicts of interest.

\section{References}

1. Kumba C, Cresci F, Picard C et al. Transfusion and Morbi-Mortality Factors: An Observational Descriptive Retrospective Pediatric Cohort Study. J Anesth Crit Care Open Access 2017 ; 8(4): 00315. DOI :10.15406/jaccoa.2017.08.00315.

2.Kumba C, Taright H, Terzi E, Telion C, Beccaria K, Paternoster G, Zerah M, Bustarret O, Jugie M, Rubinsztajn R, Treluyer JM. Blood Product Transfusion and Postoperative Outcome in Pediatric Neurosurgical Patients. EC Anaesthesia 2018; 4(8) : 288-298.

3.Kumba C, Lenoire A, Cairet P, Dogaru-Dedieu E, Belloni I, Orliaguet G. Is Transfusion an Independent Risk Factor of Postoperative Outcome in Pediatric Orthopedic Surgical Patients? A Retrospective Study. J Emerg Med Critical Care 2018 ; 4(2) :7. DOI: 10.13188/2469-4045.1000018.

4.Kumba C, Querciagrossa S, Blanc Thomas, Treluyer JM. Transfusion and Postoperative Outcome in Pediatric Abdominal Surgery. J Clin Res Anesthesiol 2018;1(1):1-8.

5.Kumba C, Querciagrossa S, Harte C, Willems A, De Cock A, Blanc T et al. A Systematic Review and Meta-analysis of Goal Directed Intra-Operative Transfusion Protocols Guided by Viscoelastic Methods and Perioperative Outcomes in Children.Int J Recent Sci Res 2019; 10 (03), pp. 31466-31471. DOI: http://dx.doi.org/10.24327/ijrsr.2019.1003.3266.

6.Kumba C, Willems A, Querciagrosssa s, Harte C, Blanc T et al. A Systematic Review and Meta- Analysis of Intraoperative Goal Directed Fluid and Haemodynamic Therapy in Children and Postoperative Outcome. J Emerg Med Critical Care 2019;5(1):1-9. D0I: 10.13188/2469-4045.1000020.

7.Kumba C, Blanc T, De Cock A, Willems A, Harte C, Querciagrossa S et al. Rapid Recovery Pathways after Surgery in Children: A Systematic Review and MetaAnalysis. Med J Clin Trials Case Stud 2019, 3(3): 000211. DOI: 10.23880/mjccs-16000211.

8.Kumba C. Transfusion in Pediatric Surgical Settings: Economic Aspects and Hospitalization Costs". Acta Scientific Paediatrics 2021 ; 4(5): 03-06. DOI: 10.31080/ASPE.2021.04.0390.

9.Kumba C. "Do Goal Directed Therapies Improve Postoperative Outcome in Children? (Perioperative Goal Directed Fluid and Hemodynamic Therapy; Transfusion goal directed therapy using viscoelastic methods and enhanced recovery after surgery and Postoperative outcome): A Study Research Protocol". Acta Scientific Paediatrics 2019 ;2(7):17-19.

Doi:10.31080/ASPE.2019.02.0094.

10.Kumba C (2020) Rationale of Goal Directed Therapies in Children. Adv Pediatr Res 7:42. Doi:10.35248/2385-4529.20.7.42. 
11. Kumba C. Physiology Principles Underlying Goal Directed Therapies in Children. Res Pediatr Neonatol.

4(4).RPN.000591.2020.Doi/10.31031/RPN.2020.04.000591.

12.Kumba C. Trans-Thoracic Echocardiographic Aortic Blood Flow Peak Velocity Variation, Distance Minute, Aortic Velocity Time Integral and Postoperative Outcome in Pediatric Surgical Patients-An Observational Pilot Study Protocol. Open Journal of Internal Medicine, 2020 ;10: $90-95$. doi:

10.4236/ojim.2020.101009.

13.Kumba C. Goal directed fluid and hemodynamic therapy and postoperative outcomes in children: Value of transthoracic echocardiographic aortic blood flow peak velocity variation: A multi-centre randomized controlled trial protocol. Adv Pediatr Res 2020; 7:35. doi: 10.35248/2385-4529.20.7.35.

14.Kumba C and Melot C. "The Era of Goal Directed Therapies in Paediatric Anaesthesia and Critical Care". EC Emergency Medicine and Critical Care 3.5 (2019): 306-309.

\section{Tables}

Table 1 General characteristics

\begin{tabular}{|c|c|}
\hline istics & $\mathrm{N}=221$ \\
\hline in months \pm standard deviation & $165.5 \pm 24.3$ \\
\hline l surgery n (\%) & $38(17.2)$ \\
\hline gery $\mathrm{n}(\%)$ & $65(29.4)$ \\
\hline ic surgery $n(\%)$ & $118(53.4)$ \\
\hline urgery n (\%) & $196(88.7)$ \\
\hline y surgery $\mathrm{n}(\%)$ & $25(11.3)$ \\
\hline y n (\%) & $15(6.8)$ \\
\hline vith intra-operative and or postoperative complications (organ failure or sepsis) $\mathbf{n}$ (\%) & $36(16.3)$ \\
\hline rative hemorrhagic shock $\mathrm{n}(\%)$ & $7(3.2)$ \\
\hline rative anaphylaxis $\mathrm{n}(\%)$ & $1(0.45)$ \\
\hline rative respiratory failure $\mathrm{n}(\%)$ & $1(0.45)$ \\
\hline tive neurologic failure $\mathrm{n}(\%)$ & $7(3.2)$ \\
\hline tive respiratory failure $\mathrm{n}(\%)$ & $5(2.3)$ \\
\hline tive cardio-circulatory failure n (\%) & $1(0.45)$ \\
\hline tive endocrinal failure $\mathrm{n}(\%)$ & $1(0.45)$ \\
\hline tive hepatic failure $\mathrm{n}(\%)$ & $1(0.45)$ \\
\hline tive renal failure $\mathrm{n}(\%)$ & $1(0.45)$ \\
\hline tive multi-organ failure $\mathrm{n}(\%)$ & $1(0.45)$ \\
\hline tive surgical wound sepsis $\mathrm{n}(\%)$ & $7(3.2)$ \\
\hline tive abdominal sepsis $\mathrm{n}(\%)$ & $5(2.3)$ \\
\hline tive septicemia $\mathrm{n}(\%)$ & $3(1.4)$ \\
\hline tive urinary sepsis $\mathrm{n}(\%)$ & $3(1.4)$ \\
\hline tive neuro-meningeal sepsis $\mathrm{n}(\%)$ & $1(0.45)$ \\
\hline tive pulmonary sepsis n (\%) & $1(0.45)$ \\
\hline l Mortality n (\%) & $1(0.45)$ \\
\hline on $\mathrm{n}(\%)$ & $83(37.6)$ \\
\hline operative hemoglobin levels \pm standard deviation $\mathrm{g} / \mathrm{dL}$ & $12.4 \pm 1.7$ \\
\hline toperative hemoglobin levels \pm standard deviation $\mathrm{g} / \mathrm{dL}$ & $11.4 \pm 1.7$ \\
\hline \%) & $7(3.2)$ \\
\hline$\%$ & $73(33)$ \\
\hline (\%) & $121(54.8)$ \\
\hline (\%) & $19(8.6)$ \\
\hline$\%)$ & $1(0.45)$ \\
\hline ngth of intensive care unit stay in days [interquartile range] & $3[1-5]$ \\
\hline ngth of hospital stay in days [interquartile range] & $5[3-11]$ \\
\hline tal length of hospital stay in days [interquartile range] & $9[5-18]$ \\
\hline ngth of mechanical ventilation (invasive or non-invasive) in days & $0[0-0]$ \\
\hline
\end{tabular}

Table 2 Surgery 


\begin{tabular}{|c|c|}
\hline Surgery & Number of patients (\%) \\
\hline Ano-rectal malformation & $1(0.45)$ \\
\hline Attached/Fixed spinal cord & $1(0.45)$ \\
\hline Cerebral cavernoma & $1(0.45)$ \\
\hline Chiari's malformation & $4(1.8)$ \\
\hline Cranioplasty & $1(0.45)$ \\
\hline Craniosynostosis & $5(2.3)$ \\
\hline Cysto-ureterectomy & $1(0.45)$ \\
\hline Decompressive craniectomy & $5(2.3)$ \\
\hline Epileptogenic lesion resection & $7(3.2)$ \\
\hline Exploratory laparotomy & $1(0.45)$ \\
\hline Femoral osteotomy & $5(2.3)$ \\
\hline Femoral prothesis & $1(0.45)$ \\
\hline Gastrectomy & $1(0.45)$ \\
\hline Gastroscopy & $1(0.45)$ \\
\hline Hepatic tumor & $2(0.9)$ \\
\hline Interscapular thoracic desarticulation & $1(0.45)$ \\
\hline Intestinal resection & 2(0.9) \\
\hline Intracerebral biopsy & $3(1.4)$ \\
\hline Intracerebral tumor resection & $28(12.7)$ \\
\hline Intraventricular stenting & $1(0.45)$ \\
\hline Laparotomy for volvulus & $1(0.45)$ \\
\hline Lefort III & $1(0.45)$ \\
\hline Limb amputation & $3(1.4)$ \\
\hline Limb tumor resection & $19(8.6)$ \\
\hline Liver transplantation & $4(1.8)$ \\
\hline Mediastinal ganglioneuroma & $1(0.45)$ \\
\hline Nissen gastrostomy & $1(0.45)$ \\
\hline Orbital tumor & $1(0.45)$ \\
\hline Pancreatectomy & $1(0.45)$ \\
\hline Pelvic tumor & $10(4.5)$ \\
\hline Polytrauma & $1(0.45)$ \\
\hline Renal transplantation & $8(3.6)$ \\
\hline Revascularisation/by-pass & $1(0.45)$ \\
\hline Scoliosis & $86(38.9)$ \\
\hline Spinal cord tumor resection & $1(0.45)$ \\
\hline Splenectomy & $2(0.9)$ \\
\hline Sterno-cleido mastoidian tenotomy & $1(0.45)$ \\
\hline Ventriculostomy & $4(1.8)$ \\
\hline Vertebral laminectomy/Arthrodesis & $2(0.9)$ \\
\hline Extradural hematoma drainage & $1(0.45)$ \\
\hline
\end{tabular}

Table 3 patient with fatal outcome

\begin{tabular}{|c|c|c|c|c|c|c|c|c|}
\hline & $\begin{array}{l}\text { Age } \\
\text { months }\end{array}$ & $\begin{array}{l}\text { ASA } \\
\text { score }\end{array}$ & $\begin{array}{l}\text { Co- } \\
\text { morbidities }\end{array}$ & $\begin{array}{l}\text { Intra-operative } \\
\text { complications }\end{array}$ & $\begin{array}{l}\text { Postoperative } \\
\text { outcome }\end{array}$ & $\begin{array}{l}\text { Delay of in-hospital mortality in } \\
\text { days }\end{array}$ & Emergency & Transfusion \\
\hline iive & 168 & 5 & Polytrauma & 0 & Multiple organ failure & 7 & Yes & No \\
\hline
\end{tabular}

Table 4 outcomes per surgery 


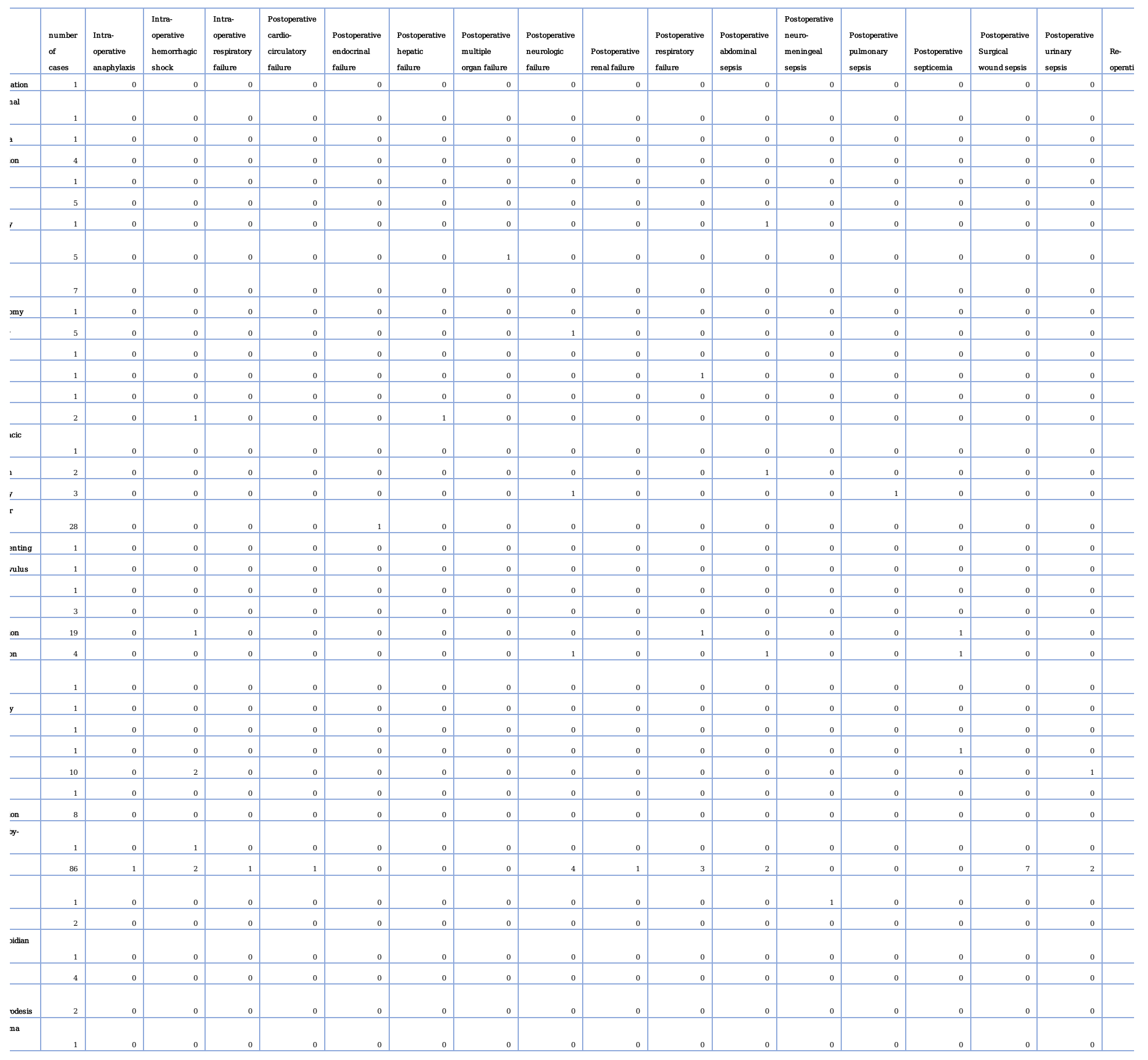

Table 5 Co-morbidities 


\begin{tabular}{|c|c|}
\hline Co-morbidity & Number of cases(\%) \\
\hline Apert syndrome & $1(0.45)$ \\
\hline Arachnoid cyst & $1(0.45)$ \\
\hline Arthritis & $3(1.4)$ \\
\hline Asthma & $1(0.45)$ \\
\hline Brain trauma & $1(0.45)$ \\
\hline Cancer & $9(4.1)$ \\
\hline Central core myopathy & $1(0.45)$ \\
\hline Cerebral anoxic lesions & $19(8.6)$ \\
\hline Chiari's malformation & $6(2.7)$ \\
\hline Chronic renal failure & $8(3.6)$ \\
\hline Congenital heart disease & $2(0.9)$ \\
\hline Convulsive encephalopathy & $1(0.45)$ \\
\hline Crouzon syndrome & $1(0.45)$ \\
\hline Di George syndrome & $2(0.9)$ \\
\hline Epilepsy & $14(6.3)$ \\
\hline Ewing's sarcoma & $12(5.4)$ \\
\hline Former pre-term with sequelae & $2(0.9)$ \\
\hline Goldenhar syndrome & $1(0.45)$ \\
\hline Gorlin's syndrome & $3(1.4)$ \\
\hline Hepatic failure & $5(2.2)$ \\
\hline Hurler's syndrome & $2(0.9)$ \\
\hline Immune deficiency & $1(0.45)$ \\
\hline Intestinal pseudo-occlusion & $1(0.45)$ \\
\hline Intracerebral tumor & $27(12.2)$ \\
\hline Intussuception & $1(0.45)$ \\
\hline Loeys-Dietz syndrome & $1(0.45)$ \\
\hline Lowe syndrome & $1(0.45)$ \\
\hline Mediastinal tumor & $1(0.45)$ \\
\hline Muscular dystrophy & $2(0.9)$ \\
\hline Myelomeningocele & $1(0.45)$ \\
\hline Neurofibromatosis & $8(3.6)$ \\
\hline Osteogenesis imperfecta & $3(1.4)$ \\
\hline Pierre Robin syndrome & $1(0.45)$ \\
\hline Xeroderma Pigmentosum & $1(0.45)$ \\
\hline Polymalformative syndrome & $2(0.9)$ \\
\hline Polytrauma & $3(1.4)$ \\
\hline Prader Wili syndrome & $1(0.45)$ \\
\hline Psychomotor deficiency & $4(1.8)$ \\
\hline Scoliosis & $1(0.45)$ \\
\hline Sepsis & $1(0.45)$ \\
\hline Spina bifida & $2(0.9)$ \\
\hline Spinal muscular a myotrophy & $1(0.45)$ \\
\hline Stroke & $1(0.45)$ \\
\hline West syndrome & $1(0.45)$ \\
\hline Wiliams syndrome & $1(0.45)$ \\
\hline
\end{tabular}

